\title{
Reformation als Frömmigkeitsbewegung: im Gespräch mit heutiger Spiritualitätsforschung
}

Kunz, Ralph

Posted at the Zurich Open Repository and Archive, University of Zurich

ZORA URL: https://doi.org/10.5167/uzh-161167

Book Section

Published Version

Originally published at:

Kunz, Ralph (2018). Reformation als Frömmigkeitsbewegung: im Gespräch mit heutiger Spiritualitätsforschung. In: Opitz, Peter. 500 Jahre Reformation: Rückblicke und Ausblicke aus interdisziplinärer Perspektive.

Berlin: Walter de Gruyter, 95-109. 


\title{
Reformation als Frömmigkeitsbewegung
}

\author{
Im Gespräch mit heutiger Spiritualitätsforschung
}

\section{Warum ist es so schwierig, fromm zu sein?}

Natürlich war die Reformation nicht nur eine Frömmigkeitsbewegung! Genauso kurzschlüssig wäre es, den Protestantismus auf ein religiöses Bekenntnis zu reduzieren und seine soziale, wirtschaftliche, politische oder kulturelle Bedeutung auszublenden. Allerdings bekommt man dann und wann den Eindruck, dass die Reformation alles sein darf, nur nicht fromm. Bundesrat Alain Berset brachte das Kunststück fertig, in einem Gastkommentar in der NZZ zum Start des Jubiläums die religiöse Dimension der Reformation komplett zu ignorieren. Er würdigte den Beitrag der Reformation als eine „Kultur des genauen Hinschauens“, eine Kultur, die sich „ohne die Geschichte der Reformation mit ihrer radikalen Diesseits-Orientierung wohl nicht erklären“" ${ }^{\text {lasse. }}$

Warum die Reformation radikal diesseitig orientiert war, sagte er nicht. Um das zu erklären, muss man genauer hinschauen, nachlesen und verstehen, wie Zwingli, Luther, Calvin und andere Gott und Welt oder Glaube und Werk unterschieden und aufeinander bezogen haben. Dann kommen wir unweigerlich auch auf die Politik zu sprechen: auf die Konflikte zwischen den Reformbewegten und den Interessen der Kurie. Aber auch die Angst vor den Saubannerzügen der kriegsverwilderten Jugend, die drohende Hungersnot oder die teilweise maroden Verhältnisse in den Klöstern trugen zur sozialen Bewegung bei, ohne die es keine Reformation der Kirche gegeben hätte. ${ }^{2}$ Fragen wir umgekehrt nach den sozialen, kulturellen und politischen Folgen der Reformation, stoßen wir auf die Idee des bonum commune ${ }^{3}$, das geistige Vermächtnis der Alten Kirche, auf eine lebendige Laien-Mystik, auf den humanistischen Bildungsoptimismus in den städtischen Eliten, den Schwung der Renaissance und das wachsende Selbstbewusstsein der

1 Gastkommentar von Bundesrat ALAIn BERSET in der NZZ am 6. März 2017: Die Aktualität reformatorischen Denkens: online: www.nzz.ch/meinung/500-jahre-reformation-die-aktualitaet-re formatorischen-denkens-ld.149016 (11.12.2017).

2 Vgl. dazu Peter Blickle: Reformation im Reich, Stuttgart ${ }^{4} 2015$.

3 Der Gemeinnutz im Gegensatz zum Eigennutz war eine wichtige Marke für das Ethos des neuen Berufs. Dazu Hans Scholl: Nit fürchten ist der Harnisch: Pfarramt und Pfarrerbild bei Huldrych Zwingli, in: Zwingliana 19, 1, Zürich 1992, 361-392. 
Zünfte in den freien Reichsstädten. Die Erfindung des Buchdrucks und die Förderung der Schulen ließen kühnste Visionen aufkommen und befeuerten endzeitliche Fantasien. Dass jeder die Bibel auf Deutsch lesen kann! - bislang eine völlig utopische Vorstellung - rückte in greifbare Nähe. Man kann das Eine nicht vom Anderen trennen.

$\mathrm{Zu}$ Beginn der Neuzeit war Religion keine Privatangelegenheit. Sie beherrschte alle Sphären des Alltags. Ihr Regiment war allgegenwärtig und beinahe allmächtig. Man konnte ihrem Anspruch nicht entfliehen - und nähme man Flügel der Morgenröte. Religion war beinahe gottgleich und darum anfällig für den Götzendienst. Es war nicht erlaubt, nicht religiös zu sein. Frömmigkeit war mit dem Leben verflochten und verwoben. Man war höchstens etwas mehr oder weniger fromm.

In der spätmittelalterlichen Gesellschaft hieß mehr religiös in der Regel, zu den religiosi und weniger religiös zu den laici zu gehören. Dabei muss man sich vor Augen halten: Rund 10\% der Bevölkerung gehörten dem geistlichen Stand an. ${ }^{4}$ Eine Untergruppe waren die saecularii. Darunter verstand man Geistliche, die keine Ordensleute waren. Diese sogenannten Welt- oder Leutpriester waren oft auch als Prediger an Prädikaturen tätig, wandten sich an das Volk und wirkten als „dirigenti religiosi“ in den Städten. Sie bereiteten gewissermaßen den geistlichen Boden für die Reformation vor. ${ }^{5}$ Denn sie riefen zur Buße, mahnten und lehrten das Evangelium, um die Frömmigkeit (pietas) der laici zu mehren. Wie jener Leutpriester namens Huldrych Zwingli, den die Chorherren am Grossmünster 1517 nach Zürich holten, um in der Kirche deutsch und deutlich zu predigen. ${ }^{6}$

Frömmigkeit ist ein Differenzbegriff. Er bezeichnet etwas Religiöses in der Religion und zielt auf eine ihr eigene Tugend und Qualität. Fromm steht für eine rechtschaffene, tüchtige, vorbildliche oder gottselige Religiosität. Sie hebt sich von einer falschen, geheuchelten oder verirrten Religion ab, wie der Glaube sich vom Aberglauben abhebt. Auf diesem Hintergrund lässt sich das Profil der reformatorischen Frömmigkeitsbewegung genauer bestimmen.

Denn mit der Unterscheidung zwischen religiosi und laici hat sie gebrochen. Folglich waren alle Pfarrer saecularii. Von einem geistlichen Stand wollten die Reformatoren ja nichts mehr wissen. Zumindest in der Theorie war es so. In der Praxis bildeten die Hirten und Diener des Wortes dann doch wieder einen geist-

4 Vgl. GEORg SCHMidt: Luther und die frühe Reformation - ein nationales Ereignis?, in: BERND MöLlER (Hg.), Die frühe Reformation in Deutschland als Umbruch, Gütersloh 1998, 54-77, 70. 5 BeRndt HAMm: Religiosität im späten Mittelalter: Spannungspole, Neuaufbrüche, Normierungen, Tübingen 2011, 391-394.

6 Ausführlicher in RALPH Kunz: Zwingli als Prediger, in: Göttinger Predigtmeditationen (GPM) 96 (2007), 119-128. 
lichen Stand. ${ }^{7}$ Gemeint war es jedenfalls anders. Folgenreicher war die starke Betonung des Glaubens, der sich im Unterschied zur Frömmigkeit nicht mehren lässt. Die theologische Kritik an der Werkgerechtigkeit hatte zur Konsequenz, dass die Dankbarkeit des Menschen die Grundlage christlicher Ethik bildet. In der Sprache des Heidelberger Katechismus tun die Menschen Gutes, weil sie dankbar sind für Gottes Wohltaten (HK 86). Die Idee einer gestuften Frömmigkeit, die zwei Stände und zwei verschiedene ethische Standards kennt, ist damit obsolet geworden. Das duale System ist Ausdruck einer falschen Religion, die Heuchelei auf der einen und Aberglauben auf der anderen Seite erzeugt. Wahre Religiosität kann nicht die Sache einer Elite sein.

Natürlich gab es schon vorher Frömmigkeitsbewegungen von und für Laien. Der Ruf in die Nachfolge war auch das Anliegen des höchst populären Büchleins Imitatio Christi von Thomas von Kempen, dem Inspirator der sogenannten devotio moderna. Frömmigkeitsgeschichtlich kann durchaus von einer Kontinuität gesprochen werden. Was Zwingli zum Thema Andacht und Gebet predigte und schrieb, war stark beeinflusst von der niederrheinischen Mystik. Zur eigenständigen Frömmigkeitsbewegung wurde die Reform der Kirche aufgrund verschiedener Faktoren, die im Verbund zu Synergien wurden. Der Impuls, den hierarchischen Aufbau der Bischofskirche zu hinterfragen und der Anspruch, das gottselige, ehrbare und aufrichtige Leben zur kollektiven Norm zu erheben, wirkten zusammen, warfen aber in der institutionellen Umsetzung auch einen Schatten. Denn die Kombination eines so hohen individuellen Ethos mit einem Programm der kollektiven Durchsetzung führte notwendigerweise zu Konflikten mit denen, die frömmer als nur fromm oder nicht immer so fromm sein wollten also mit den Täufern auf der einen und mit den gemeinen Leuten auf der anderen Seite.

Es sei dem Praktischen Theologen erlaubt, die historisch höchst diffizile Ausdifferenzierung und Ausbalancierung des Glaubenssystems holzschnittartig zu vereinfachen. Darum geht es mir, zu zeigen, dass sich das Wörtchen fromm als ein schwieriger Freund erweist. Unsere fromme Seele ahnte es, als wir bei der letzten Gelegenheit wieder einmal die Landeshymne gesungen haben. ${ }^{8}$ Ist fromm ein Gefühl, das sich nur noch beim Morgenrot einstellt? Selbst Kirchgänger stut-

7 Vgl. Hans Scholl: Pfarramt und Pfarrerbild (wie Anm. 3), 390. Zur Entwicklung vgl. RaLPH KunZ: „Ohn Habit und Kragen“ - vom Kerngeschäft im Pfarramt, in: JAN BAUKE/MATTHIAS KRIEG (Hgg.), denkMal, Bd. 6, Zürich 2003, 31-49.

8 Zur Frömmigkeit der Landeshymne vgl. RALPH KunZ: Der Bettag als Busstag. Von der Aktualität eines Brauchs, in: Eva-Maria Faber/Daniel Kosch (Hgg.), Dem Bettag eine Zukunft bereiten. Geschichte, Aktualität und Potential eines Feiertages, Zürich 2017, 225-240. 
zen, wenn sie im Gesangbuch mit Johann Heermann (1585-1647) singen: „O Gott Du frommer Gott, Du Brunnquell aller Güter.“

Offensichtlich hat sich semantisch etwas verschoben. Das althochdeutsche fruma und mittelhochdeutsche frumb, das sich aus primus ableitet, meinte vorbildlich, rechtschaffen und nützlich. In der altertümlichen Wendung „es frommt“ klingt diese Bedeutung noch an. ${ }^{9}$ Später wurde daraus etwas Gefühltes und noch später etwas Verkrampftes. Paradoxerweise ist ausgerechnet die starke Betonung des Glaubens dafür verantwortlich. Schon Luther konnte fromm im Sinne von gottselig verwenden, also auf eine Emotion verweisen. Das Pathos schob sich über das Ethos, was der Reputation der Frommen nicht sonderlich gut bekam. Wenn man die fragt, die im Ernst nicht Christen sein wollen, klingt fromm nach frömmlerisch, also: selbstgerecht, heuchlerisch, missionarisch, fanatisch, sektiererisch und gefährlich. Wer noch eins draufsetzen möchte, fügt hinzu: freikirchlich, pharisäisch und fundamentalistisch.

Offensichtlich hat sich auch in der Wertung etwas verschoben. Was ist passiert? Wer genauer hinschaut und wissen will, was es mit der Wirkungsgeschichte der reformatorischen Frömmigkeit auf sich hat, hält sich am besten an Historiker, die Frömmigkeit sozusagen mit dem Fernrohr beobachten. Den Praktischen Theologen interessiert die Gegenwart.

\section{Warum taugt der Kunstbegriff Spiritualität doch zu etwas?}

Tatsache ist, dass das Frommen-Bashing heute salonfähig ist. Man kann damit punkten. Dass mit denen, die frömmer sein wollen, etwas nicht stimmen kann, gehört zur Verdachtshermeneutik der Moderne. Und dass man es nicht nötig hat, am Sonntag in die Kirche zu springen, ist ein Bekenntnis, das auch Mitglieder der Kirche ohne Schamröte aufsagen. Eine Analyse unseres säkularen Zeitalters würde den Rahmen dieses Beitrags sprengen. Sie könnte bei Immanuel Kant starten und bei Peter Sloterdijk landen. Sie müsste Peter Berger und Charles Taylor zitieren und käme dann zum Schluss, dass die Frömmler-Kritik ein Phä-

9 Vgl. Artikel „fromm“, in: JACOB GRIMm/WiLheLm GRIMm (Hgg.), Deutsches Wörterbuch von Jacob Grimm und Wilhelm Grimm, Erstbearbeitung (1854-1960), digitalisierte Version im Digitalen Wörterbuch der deutschen Sprache: www.dwds.de/wb/dwb/fromm (12.12.2017). 
nomen ist, das sehr eng mit der Geschichte des europäischen Christentums zusammenhängt. ${ }^{10}$

Für genauso wichtig halte ich die Beobachtung, dass diese Kritik jede Frömmigkeitsbewegung begleitet. Die Frommen wollen keine Frömmler sein. Es gehört in gewisser Weise zum Programm der Frömmigkeit, dass die übertriebene, selbstgerechte, auf den eigenen Auftritt bedachte, genauso wie die devote oder die bigotte Religiosität wenig mit Gottvertrauen zu tun hat. Diese kritische Differenz finden wir auch bei Jesus und bei den alttestamentlichen Propheten. Allerdings wird Religion nicht in Bausch und Bogen verworfen oder als Übel angesehen. Die fromme Frömmler-Kritik verlangt nach der Unterscheidung einer wahren und falschen oder gesunden und kranken Religiosität. Ums Unterscheiden kommt man nicht herum, was eine Referenz nötig macht. Woran misst man die gesunde Frömmigkeit? Wann wird aus dem frömmlerischen Wesen eine Krankheit?

Eine psychologische Analyse der gesunden Religiosität würde den Rahmen dieses Beitrags sprengen. Sie könnte bei William James starten und bei Tilman Moser landen. Sie müsste auf die Entwicklungen der Religionspsychologie verweisen und käme dann zum Schluss, dass jeder Versuch, einen neutralen Standpunkt einzunehmen, scheitern muss. Denn wir fragen, wenn wir nach Religion fragen, immer mit der Vorgabe eines gewissen Ideals, ob wir dieses nun mit Gesundheit oder Wahrheit in Verbindung bringen.

An dieser Stelle gibt es einen Berührungspunkt zur Spiritualitätsforschung. Man hört es schon am Klang. Wer nach Spiritualität fragt, fragt nach dem, was frommt im ursprünglichen Sinne. Wer der religiösen Enge entfliehen möchte, benutzt aber besser nicht das fromme Vokabular. Wer sich nicht verdächtig machen möchte, lässt sich inspirieren, begeistern, berühren oder erschüttern. Man vermeide um Himmels willen Gott und rede lieber von Engeln.

Spirituell steht für Echtheit. Und ich sage es gleich vorweg: Wir handeln uns mit der Echtheitsvermutung neue Probleme ein. ${ }^{11}$ Denn wenn Frömmigkeit damit zu kämpfen hat, dass sie zu eng verstanden wird, hat man es bei der Spiritualität mit einer zu großen Weite zu tun. Frömmigkeit ist vom Zeitgeist schon beinahe entsorgt, Spiritualität ist ein Begriffscontainer, mit dem derselbe Geist fast alles besorgen kann. Um es mit dem Berliner Philosophen Byung-Chul Han zu sagen: Während Frömmigkeit rau ist, bleibt Spiritualität glatt. ${ }^{12}$ Sie passt sich an. Es gibt sie in allen Varianten: religiös und areligiös, körperbetont oder ganz vergeistigt.

10 Eine gut lesbare Zusammenfassung der Säkularisierungsdebatte findet sich bei ALEXANDER GARTH: Gottloser Westen?, Leipzig 2017, 44-80.

11 GARTH, Gottloser Westen? (wie Anm. 10), 81-86.

12 Byung-Chul Han: Die Errettung des Schönen, Frankfurt am Main 2015. 
Man liebt sie bei Greenpeace, im Silicon Valley und an der Wallstreet. Ihr Marktwert ist hoch, aber ihr Ruf bleibt schillernd.

Das alles weiß ich und halte dennoch an beiden Begriffen fest. Denn ich verspreche mir etwas davon, das Raue neben das Glatte und das Dichte neben das Weite $\mathrm{zu}$ stellen und es $\mathrm{zu}$ vergleichen. Zumal Verbindungen und Kreuzungen erkennbar sind. Sie lassen sich anhand der Begriffsgeschichte von Spiritualität zeigen. Simon Peng-Keller hat sie herausgearbeitet und konnte aufzeigen, welche faszinierenden Umwege der Begriff gemacht hat. ${ }^{13}$ Es scheint fast so, dass der Niedergang der Frömmigkeit mit dem Aufstieg der Spiritualität zusammenfällt. Zur Attraktivität der Spiritualität trägt bei, dass dem Begriff ein Bild zugrunde liegt. Spiritus ist die Übersetzung der hebräischen Ruach und des griechischen Pneuma. Man spürt den Atem und den Wind und man sieht die Flammen, die nicht verbrennen und das Wasser, das nicht versiegt.

Von den biblischen Wurzeln her betrachtet ist Spiritualität das Leben des Geistes im Menschen und Spiritualitätsforschung eine Aufgabe der Theologie. Ziel ist es, das Wirken des Geistes als Formationsprinzip und Expression zu erforschen und das meint, sowohl das geschichtlich Gewachsene wie auch das Verwachsene auf diesem Hintergrund zu verstehen und auf dieser Grundlage

- prinzipiell nach dem spirituellen Wachstum zu fragen, das in der Dialektik von Rechtfertigung und Heiligung entstehen kann,

- material über Praktiken nachzudenken, die den Menschen helfen können, sich auf die Reich-Gottes-Bewegung einzulassen

- und formal zu prüfen, in welchen Gefäßen Einzelne und Gemeinschaften geistlich begleitet werden.

Mit anderen Worten: die Praktische Theologie ist fromm. Sie hat eine Sendung und vertritt einen engagierten Ansatz der Forschung. Sie will etwas verändern. Sie ist eine Praxistheorie, die im Dreischritt Sehen - Urteilen - Handeln Wirkung erzielen möchte.

Das ist gegen ein verengtes Wissenschaftsverständnis gesagt, das nur die Deskription als wissenschaftlich gelten lässt, aber genauso gegen ein verengt quietistisches oder privatisiertes Religionsverständnis gerichtet, das Religiöses nur unter Erfahrung abhandeln will. Praktische Theologie ist nicht religiöse Praxis. Sie reflektiert die Differenz von Leben und Lehre. Aber sie könnte ihrer Aufgabe nicht gerecht werden, wenn sie einen Bruch zwischen Praktischer

13 Simon Peng-KelLer: Spirituelle Erfahrung als locus theologicus. Theologische Reflexion auf gelebte christliche Spiritualität, in: Eva-MARIA FABER (Hg.), Lebenswelt und Theologie. Herausforderungen einer zeitsensiblen theologischen Lehre und Forschung, Fribourg 2012, 261-292. 
Theologie und theologischer Praxis zulassen würde. Sie sucht das Gespräch mit dem Erbe der Reformation, um daraus etwas für die Spiritualität der Gegenwart zu lernen.

\title{
3 Reformation als Anleitung zum Frommwerden?
}

Wenden wir uns also noch einmal der Reformation als Frömmigkeitsbewegung zu und reden von denen, die „mit Ernst Christen“ sein wollen. So hat Martin Luther die Zielgruppe der Reform 1526 in der Vorrede zur Deutschen Messe umschrieben. Er unterscheidet drei Weisen des Gottesdienstes: die lateinische Messe zur Schulung der Jugend, die Deutsche Messe zur Unterweisung des Volkes und die dritte Weise. Er schreibt dazu:

\begin{abstract}
Die dritte Weise, welche die rechte Art der evangelischen Ordnung haben sollte, [sie] dürfte nicht so öffentlich auf dem Platz unter allerlei Volk geschehen. Sondern diejenigen, die mit Ernst Christen sein wollen und das Evangelium mit der Tat und dem Munde bekennen, müßten sich mit Namen einzeichnen und sich etwa in einem Haufen versammeln zum Gebet, lesen, zu taufen, das Sakrament empfangen und andere christliche Werke zu üben. In dieser Ordnung könnte man die, welche sich nicht christlich hielten, kennen, strafen, bessern, ausstoßen oder in den Bann tun nach der Regel Christi (Matth. 18,15ff). Hier könnte man auch ein gemeinsames Almosen auferlegen, das man freiwillig gäbe und nach dem Vorbild des Paulus austeilte (2. Kor. 9,1). Hier bedürfte es nicht vieler und großer Gesänge. Hier könnte man auch Taufe und Sakrament auf eine kurze feine Weise halten und alles aufs Wort und Gebet und auf die Liebe richten. Hier müßte man einen guten kurzen Unterricht über das Glaubensbekenntnis, die zehn Gebote und das Vaterunser haben. In Kürze: wenn man die Menschen und Personen hätte, die mit Ernst Christen zu sein begehrten, die Ordnungen und Regeln dafür wären bald gemacht. ${ }^{14}$
\end{abstract}

Luthers „dritte Weise“, das wird aus dieser Beschreibung ersichtlich, ist ein Ziel einer Reform, das 1526 noch nicht erreicht war. ${ }^{15}$ Er selbst schrieb: „Ich habe die Leute noch nicht. “16 Er fand sie auch später nicht.

Tatsächlich kämpfte Luther sein Leben lang mit dem Gefühl des Scheiterns. Das ist zum einen Stoff für Lutherbiografien, wie sie die Oxforder Historikerin

14 MARTIN LUTHER: Vorrede zur deutschen Messe (1526), in: KARIN BORNKAMm/GERHARD EBeLING (Hgg.), Martin Luther. Ausgewählte Schriften. Kirche, Gottesdienst, Schule, Frankfurt am Main [u.a.] 1995, $77 \mathrm{f}$.

15 Sybille RolfF: „Das Evangelium mit Taten und Worten bekennen“. Die Kommunikation des Evangeliums im Anschluss an reformatorische Theologie, in: Christiane Moldenhauer/Jens Monsees, Die Zukunft der Kirche in Europas, Neukirchen-Vluyn 2016, 1-19, 11.

16 LuTHER, Vorrede (wie Anm. 14), $77 \mathrm{f}$. 
Lyndal Roper vorlegte. ${ }^{17}$ Ihre akribischen Studien der Quellen zeichnen ein Bild der Person Luthers, die fasziniert und erschreckt: sie zeigen das Psychogramm des Seelsorgers und Theologen, aber auch den Antisemiten, den Choleriker, den Lebemenschen, Apokalyptiker und Hexenverfolger, der mit seiner Kirche haderte. Aber Wittenberg ist nicht Zürich und Zürich nicht Genf. Die Reformation im Norden, im Süden und im Westen ging unterschiedliche Wege. Das hat zum einen mit den Protagonisten zu tun und zum anderen mit den politischen Verhältnissen. In der kommunalen Reformation konnte sie lokaler und kleinräumiger Fuß fassen. Die Differenzen zeigten sich in der Abendmahlstheologie, in der Ekklesiologie, der Christologie und der Soteriologie - bald wurden daraus tiefe Risse und erbitterte Fehden.

Ich werde mich hüten, diese Unterschiede klein zu reden. Sie sind Stoff für theologische Seminare, akribische Studien und subtile Schulbildungen. Wenn ich das Gemeinsame hervorhebe, hat es mit dem erkenntnisleitenden Interesse an einer evangelisch inspirierten Frömmigkeit zu tun. Bei allen Unterschieden verbindet Luther, Zwingli und Calvin dieselbe evangelische Frömmigkeit. Es ist kein Zufall, dass Martin Luther in seiner Vision einer „dritten Weise“ der christlichen Ordnung dem Ideal der reformierten Konzentration sehr nahekommt:

Hier bedürfte es nicht vieler und großer Gesänge, hier könnte man auch Taufe und Sakrament auf eine kurze feine Weise halten und alles aufs Wort und Gebet und auf die Liebe richten. Hier müßte man einen guten kurzen Unterricht über das Glaubensbekenntnis, die zehn Gebote und das Vaterunser haben. ${ }^{18}$

Was Luther in Wittenberg nicht gelingen wollte, versuchte man in Zürich und Genf inklusive Kirchenzucht umzusetzen. Und es ist sicher auch kein Zufall, dass sich rund hundertfünfzig Jahre später Philipp Jacob Spener in seinen Schriften auf die Vorrede Luthers berief. In der Pia Desideria beklagt er den Mangel an wahrem, lebendigem Glauben, vor allem im geistlichen Stand. ${ }^{19}$ Der Vater des Pietismus knüpft beim Reformator an, zielt aber mit seinem Programm der Erneuerung auf das Priestertum aller Gläubigen, auf den Haufen, der sich versammelt, auf das, was er später ecclesiola in ecclesia (1675) und collegium pietatis (1677) nannte.

17 Lyndal Roper: Der Mensch Martin Luther. Die Biografie, Frankfurt am Main 2016.

18 LUTHER, Vorrede (wie Anm. 14), $77 \mathrm{f}$.

19 Die Pia desideria (mit dem Untertitel: Herzliches Verlangen nach gottgefälliger Besserung der wahren evangelischen Kirche) ist der Titel einer 1675 erschienenen Schrift von PHILIPP JAcoB SPENER: Pia Desideria, hg. von KuRT ALANd (= Kleine Texte für Vorlesungen und Übungen, Nr. 170). Berlin ${ }^{3}$ 1964. Vgl. dazu MARTIN BRECHT: Philipp Jakob Spener, sein Programm und dessen Auswirkungen, in: DeRs. (Hg.), Geschichte des Pietismus, Bd. 1, Göttingen 1993, 302-316. 
Auch bei Spener lassen sich reformierte Anliegen erkennen: der Nachdruck auf der Heiligung, das große Gewicht der Versammlung der Bekennenden und die Mahnung zur tätigen Liebe. Und der Pietismus kämpft auch mit denselben Schatten: der Tendenz zum Kongregationalismus, der Gesetzlichkeit und der Weltfremdheit. Ein Vergleich mit dem Puritanismus, dem Methodismus und dem Evangelikalismus drängt sich auf.

Das ist Stoff für die Kirchengeschichte. Der einfach gestrickte Praktische Theologe begnügt sich mit der Feststellung, dass sich in der evangelischen Frömmigkeit zwei starke Bewegungsimpulse finden lassen: der Impuls, wieder beim Anfang anzufangen und der Impuls, nach dem geistlichen Wachstum zu streben. Beide Impulse brauchen und beobachten einander. Vielleicht ist es gar simpel, aber hilfreich um die Dynamik zu verstehen, wenn wir den einen Pol mit der Freiheit des Christenmenschen und den anderen mit dem Gehorsam verbinden. ${ }^{20}$ Diese Dynamik beruht auf einer Dialektik, die unruhig macht und in Bewegung setzt. Denn der wahre Fromme weiß, dass sich der Anfang bei der Gnade einem Ruf verdankt, der auf den Weg der Nachfolge schickt, der Heiligung heißt und zu einer praxis pietatis führt, die von Paulus auch Wandel im Geist genannt wird. Es ist ein Weg des Einzelnen und ein Weg der Kirche, ein Weg zwischen den Zeiten unter dem Zeichen des Vorletzten - und immer auch ein Unterwegssein Gottes mit uns.

Ein Ende ist erst dann in Sicht, wenn aus dem Glauben ein Schauen und Gott alles in allem sein wird. Also ist vorläufig kein Ende in Sicht. Darum bleibt die Reformation eine Frömmigkeitsbewegung. Genau das meint die arg strapazierte Formel ecclesia semper reformanda in der Essenz. In einer spannenden Spurensuche ihrer Wirkungsgeschichte kommt Emidio Campi zum Schluss, die Formel wolle letztlich sagen, „dass die Christenheit immer wieder von neuem hat beginnen können, dort, wo es ihr gelungen ist, Wege frei zu halten zur Begegnung mit der Kraft und der Herrlichkeit des Wortes vom Kreuz, das Verwandlung ermöglicht, zur Umkehr einlädt und zur Mitgestaltung einer humaneren Gesellschaft einlädt.“21

Das ist fromm gesagt und bringt schön auf den Punkt, woher die Kraft der Erneuerung kommt. Von einem göttlichen Gegenüber, das in einer anfänglichen

20 Um wieder an ein berühmtes Lutherwort zu erinnern: „Ein Christenmensch ist ein freier Herr über alle Dinge und niemand untertan. Ein Christenmensch ist ein dienstbarer Knecht aller Dinge und jedermann untertan.“, aus: MARTIN LuTHER, Von der Freiheit eines Christenmenschen (1520), Gütersloh 2006. Vgl. dazu: ReInHold RIEGER: Von der Freiheit eines Christenmenschen, De libertate christiana, Tübingen 2007, 5-12.

21 Emidio CAmpi: „Ecclesia semper reformanda“. Metamorphosen einer altehrwürdigen Formel, in: Zwingliana 37 (2010), 1-20, 19. 
Begegnung in, mit und durch das Wort erfahren wird. Deshalb propagierten die Reformatoren eine durch das Wort Gottes und nicht durch ihre Frömmigkeit erneuerte Kirche - was selbstredend dem Geist dieser Frömmigkeit zugeschrieben werden kann. Das mag spitzfindig erscheinen, ist es aber nicht. Denn man kommt nur dann zur Kraft der Reformation, wenn man diese radikalste aller möglichen Selbstunterscheidungen, die in ihrer Frömmigkeit angelegt ist, nicht überspielt. Die Bewegung der Frömmigkeit ist eine Bewegung zu Gott, also Buße, angestoßen durch Gott, also Heiligung. Sie wird theonom realisiert: Die wahre Frömmigkeit verlässt sich nicht auf reine Tugend, religiöse Erregung oder durchdachte Lehre. Aber sie wird reziprok aktualisiert: Der Mensch sucht Gott, hält an seiner Gerechtigkeit fest und verlässt sich auf seine Treue. Als frommer Mensch weiß er zwischen dem Heiligen Geist und der eigenen Begeisterung zu unterscheiden und erfährt diese Differenz als Krisis und Verheißung seiner christlichen Existenz. ${ }^{22}$

Man kann diese Differenz bis ins Extrem der gedanklichen Paradoxie treiben und hätte dann die Pointe der Spiritualität schon wieder verpasst. Nicht um spitzfindige Aporien oder paradoxale Lebenskonzepte geht es hier; auch nicht um die Illusion einer totalen Übereinstimmung von Lehre und Leben, sondern um die widerständige Praxis einer Lebensform, die dem Sog der Selbstbehauptung zu entgehen sucht. Es ist die Funktion rechtverstandener Rechtfertigung, den religiösen Eigensinn der christlichen Lebenspraxis zu erhalten. Denn wir sind's noch nicht, wir werden's aber. Geben wir noch einmal Martin Luther das Wort:

Das Leben ist nicht ein Frommsein, sondern ein Frommwerden, nicht eine Gesundheit, sondern ein Gesundwerden, nicht ein Sein, sondern ein Werden, nicht eine Ruhe, sondern eine Übung. Wir sind's noch nicht, wir werden's aber. Es ist noch nicht getan oder geschehen, es ist aber im Gang und im Schwang. Es ist nicht das Ende, es ist aber der Weg. Es glüht und glänzt noch nicht alles, es reinigt sich aber alles. ${ }^{23}$

22 Der Heilige Geist wird zur Krisis der Theologie, wenn sie sich nicht auf die Vollendung der Schöpfung hin entfaltet. Rudolf BoHren: Dass Gott schön werde. Praktische Theologie als theologische Ästhetik, München 1975, 18.

23 MARTIN LutheR: Grund und Ursach aller Artikel (1521), Werke. Kritische Gesamtausgabe, Weimarer Ausgabe (WA), Schriften 7, Weimar 1897, 309-457, 336. 


\section{Was kann die Spiritualitätsforschung Erhellendes zum Verständnis der Reformation beisteuern?}

Was hat das mit Spiritualitätsforschung zu tun? Der Begriff ist erklärungsbedürftig und begründungspflichtig, gerade weil er so cool daher kommt, glatt und nicht rau ist. Was ist darunter zu verstehen? Und was unterscheidet Spiritualität von Glauben, Religiosität oder Frömmigkeit? Die Frage liefert Stoff für interdisziplinäre Seminare. Als einfach gestrickter Praktischer Theologe erlaube ich mir, mit einer pragmatischen Unterscheidung weiterzuarbeiten. Spiritualität kann weit gefasst werden. In den Gesundheitswissenschaften und in der Psychologie kursieren Definitionen einer frei flottierenden, fluiden und flüchtigen Orientierung, die nach Sinn, Verbundenheit und Transzendenz fragt. In der Religionswissenschaft wird Spiritualität auch im Sinne einer Alternative zur Religiosität verwendet.

Den theologischen Diskurs bringt eine solche Begriffsextension aus naheliegenden Gründen in Verlegenheit. Wer danach fragt, wie Christen in der Gegenwartskultur ihren Glauben leben, oder wie sie Praktiken einüben, die ihnen helfen, ihr Leben zu führen, oder welche Antworten sie auf die großen Fragen der Zeit haben, oder was sie tun, um glaubwürdig das Evangelium zu kommunizieren - wer nach all diesen Dingen fragt, interessiert sich für eine Intensivierung, Vitalisierung und Erneuerung seiner Religion und nicht für ihre Auflösung. Dieses Ansinnen könnte der Theologie den Vorwurf der Enge einbrocken. Die fromme Spiritualität ist aber nicht eng, nur weil sie nicht zu weit sein will. Engführung ist nicht ihr Ziel und Engstirnigkeit ist ihr ein Gräuel. Evangelische Spiritualität will Tiefe! Man ahnt in der Bewegung zu den Tiefen Gottes das Geheimnis, das man nur umkreisen kann (1. Kor 2,10) und stößt in der Bewegung nach oben an die Grenzen der Erkenntnis, die weiß, dass man darüber hinaus nicht denken kann.

Aber was beweist das? ${ }^{24}$ Womit prüfen wir die Gesundheit, Wahrheit oder Echtheit des religiösen Erlebens? Raummetaphern sind trügerisch. Von Höhen und Tiefen zu reden, könnte auch ein Trick sein. Das Tiefe kann abgründig und das Hohe übermütig werden. Auch ein raffiniertes Sprachspiel, das sich auf der Klaviatur der Gefühle auskennt, erzeugt nur relative Evidenz. Man muss genauer hinschauen und sich einlassen auf die Logik des Glaubens, die auf ein Verhältnis

24 In Anspielung auf den ontologischen Gottesbeweis nach Anselm. Vgl. dazu BURKHARD MoJSIsch: Anselm von Canterbury. Gottesbeweise, in: Tнео Ковusch (Hg.), Philosophen des Mittelalters. Eine Einführung, Darmstadt 2000, 42-53. 
zielt. Ohne Bezug auf die Gottesbeziehung ist Frömmigkeit nicht zu denken. Wenn wir verstehen wollen, was die Reformation bewegt hat und in Bewegung bringen wollte, rasseln wir mit Formeln wie radikaler Diesseitsorientierung voll ins Abseits. Dafür stehen die Höhe und die Tiefe. Wenn ich die Vertikale im Raum die mystische Achse nenne, nehme ich das Risiko eines gefährlichen Begriffs in Kauf.

Ich will es am Beispiel von Zwinglis Abendmahlstheologie illustrieren. ${ }^{25}$ Diese lässt sich nicht verstehen ohne eine Formel, die er von Augustin übernimmt. Das Nachtmahl sei eine Danksagung, eine geistliche Speise, bei der Gott genossen werde. Das ist Mystik mit einem klaren biblischen Bezugspunkt. Gott zu lieben ist das erste Gebot. Der Begriff des Genusses macht auf die entscheidende Front aufmerksam. Gott soll weder benutzt oder verwendet noch soll Religion anstelle Gottes verehrt werden.

Allein Gott in der Höhe sei Ehre ist eine spirituelle und keine moralische Regel. Der zur Anbetung freie Mensch ist von den Zwängen des Götzendienstes befreit. Ohne Genuss-Metaphorik lässt sich die reformatorische Frömmigkeitsbewegung gar nicht verstehen. Ohne Freude an Gott wäre sie keine Bewegung. Ohne Glauben wäre sie nur noch Religion. Ohne Spiritualität würde sie zu jenem Zerrbild, das religiös unmusikalische Kritiker aus ihr gemacht haben. Ohne die Freiheit des Christenmenschen wird aus dem Frommen der verklemmte puritanische Frömmler, der sich nicht getraut, das Leben zu genießen.

Ein Gespräch über die reformatorische Frömmigkeitsbewegung, das von seiner mystischen Seite nichts wissen will, endet in der Regel mit diesem Klischee. Sie kann nicht durch eine soziologische oder psychologische Aufklärung religiöser Verformung ersetzt werden. Sie darf sich nicht von ihrer Spiritualitätsforschung abhalten lassen, die die Frömmigkeit auch mit einer Vertrauenshermeneutik befragt. Weil sie den Segen, der daraus erwächst, sieht und weil sie das Zeugnis der Menschen, die ihren Glauben treu gelebt haben, hört. Weil sie will, dass Gott schön wird.

\section{Warum sollt ich meinem Gott nicht singen?}

Damit ist neben der Gesundheit, der Wahrheit und der Echtheit zum Schluss auch die Schönheit der Religion ein Thema. Gott genießen und die Welt verändern, Beten und das Gerechte tun - darauf läuft es hinaus - geht nicht ohne Schönheit. Sie hält den Glauben in Bewegung. Natürlich verändert sich die Stimmung in der

25 Ausführlicher in: RALPH KunZ: Abendmahlsgottesdienst, in: DAVID Plüss [u.a.] (Hg.), Gottesdienst in der reformierten Kirche. Einführung und Perspektiven, Zürich 2017, 224-242. 
Weggemeinschaft der Gläubigen. Diese ist nicht immer schön. Sie ist manchmal düsterer und manchmal heller, manchmal kämpferischer und manchmal kontemplativer. Die Stimmungen ändern sich, je nachdem, was dran ist. So geht das $\mathrm{zu}$ in Bewegungen. Aber das Ganze hat einen Glanz, den man sehen kann und eine Kraft, die man spüren kann, wenn man sich darauf einlässt.

Darum geht mir diese ewig dumme Phrase von den lustfeindlichen Zwinglianern auf die Nerven. Es ist das Stroh, das halbgebildete Möchtegernexperten dreschen, weil sie keine Ahnung von der hundertfältigen Frucht des Geistes haben. Halten wir ihnen entgegen und bezeugen die Freude, die aus der Leidenschaft kommt, die Lust am Glauben, die den Gehorsam nicht verweigert und die Begeisterung, die den Schmerz nicht ausspart. Das ist heute dran! Die Spiritualität der evangelischen Frömmigkeit hat Tiefgang und bietet sogar einmal einen Höhenflug. Ich würde deshalb, anders als Martin Luther im Nachwort zu seiner Vorrede, denen, die mit Ernst Christen sein wollen, diejenigen zugesellen, die es mit Freude sein wollen. Weil sie etwas zu sagen und zu singen haben. ${ }^{26}$

Darum komme ich zum Schluss noch einmal auf den Anfang zurück. Der Redenschreiber von Alain Berset hat seine Hausaufgaben gemacht. Er hat Max Weber gelesen und verstanden, dass die Hinwendung zur Welt und das Zeugnis mit Tat und Mund eines jeden Christen in seinem Beruf und Stand ein zentrales Motiv der Reformation war. Aber ohne Musik würde aus dieser radikalen Diesseitsorientierung etwas ganz und gar Ernstes. Was fehlt, ist die Aussicht auf ein glorioses Ende, eine Aussicht, die keine ist und darum einen Blick schenkt für das, was sich jetzt schon fragmentarisch erkennen, schmecken und sehen lässt.

Was wir jetzt schmecken, ist ein Vorgeschmack auf den Genuss Gottes, das höchste Glück, über das hinaus man kein Glück genießen kann. Die zeitgenössische Spiritualität zieht es vor, von kosmischer Tiefe oder dem Wunder des Universums zu sprechen. Es macht keinen Sinn, darüber zu streiten, wann es besser ist, rau oder glatt $\mathrm{zu}$ reden. Wichtiger ist die Emotion, die immer noch funkt, glüht und glänzt durch alle Jahrhunderte hindurch und die Frage, wo und wie sich dieses Funken, Glühen und Glänzen finden lässt. Die Schönheit gibt einen Fingerzeig.

John Eliot Gardiner hat über Johannes Sebastian Bach ein siebenhundertseitiges Opus mit dem Untertitel Musik für die Himmelsburg geschrieben. Es ist eine dichte Beschreibung von Person und Werk, bei der man viel über die

26 Die Betonung eines evangelischen Propriums will nicht übersehen, dass die Musik auch ein Medium der ökumenischen Einheit darstellt. Vgl. dazu FLORIAN IHSEN: Eine Kirche in der Liturgie. Zur ekklesiologischen Relevanz ökumenischer Gottesdienstgemeinschaft, Göttingen 2010, 60 170. 
Schönheit lernen kann und es ist zugleich das Bekenntnis zu einer Spiritualität, in der Pathos, Ethos und Logos zu einer musikalischen Gestalt zusammenfinden:

\begin{abstract}
Seine [scil. Bachs, RK] Musik offenbart seinen tiefen Abscheu vor Heuchelei und seine Unduldsamkeit gegenüber jeglicher Verfälschung von Tatsachen, aber auch sein tiefes Mitgefühl für alle, die in irgendeiner Form leiden müssen, betrübt sind oder von Gewissensbissen oder Glaubenszweifeln geplagt werden. All das veranschaulicht seine Musik, und daraus bezieht sie ein Gutteil ihrer Authentizität und ihrer ungeheuren emotionalen Wucht. Vor allem aber macht sie hörbar, mit welcher Freude und mit welchem Vergnügen er die Wunder des Universums und die Geheimnisse des Lebens feierte - und in seiner eigenen schöpferischen Gewandtheit schwelgte. Man muss sich nur eine einzige Weihnachtskantate anhören, um Musik von einer jauchzenden, festlichen Begeisterung zu erleben, an die kein anderer Komponist herankommt [...] Viele von uns können angesichts dessen nur staunen, in sich gehen und vor Gedankengängen kapitulieren, deren Spiritualität so tiefgründig und unwandelbar ist wie die kaum einer anderen Musik. ${ }^{27}$
\end{abstract}

So redet einer, der sein Leben der Musik gewidmet hat, über einen, der seine Musik Gott geweiht hat - im Wissen, dass Bach von der Aussicht beseelt war, „nach dem Tod ein besseres Leben in der Gemeinschaft von Engeln und engelsgleichen Musikern zu verbringen. “28 Wir wollen Herrn Gardiner deshalb verzeihen, dass sein soli deo gloria manchmal zu einem Solo für den frommen Bach wird.

Die Lektüre will nicht zu einer Bachverehrung führen, sondern von Bach lernen, dass sich das erste Gebot im Lob verwirklicht. Das anfängliche Motto der biblischen Spiritualität ist auch ihre letzte Aussicht. Alles, was atmet, lobe den Herrn. Gottes Ehre lässt den Menschen nicht verstummen, sondern weckt die Lust, einzustimmen in eine kosmische Symphonie. Der Mensch ist geschaffen, um zu jubilieren. Das ist eine Botschaft, von der man singen und sagen muss. Der Westminster Shorter Catechism formuliert wunderbar prägnant den reformierten Nachdruck auf diese Bestimmung der menschlichen Existenz. Das Bekenntnis antwortet auf die erste Frage, was denn diese Bestimmung sei, mit dem ebenso schön wie knapp formulierten Grundsatz: „Man's chief end is to glorify God, and to enjoy him forever. “29

Die Reformierten haben eine Ahnung davon, dass das Singen Kopf und Herz verbindet und wie sich der himmlische im irdischen Gottesdienst anfühlt. Jean

27 John Eliot GaRdineR: Bach. Musik für die Himmelsburg, München 2016, 30 f.

28 GARDINER, Bach (wie Anm. 27), $30 \mathrm{f}$.

29 Der Westminster Shorter Catechism ist zu finden unter: www.reformed.org/documents/WSC. html (12.12.2017). 
Calvin, der Gesang und Musik in den höchsten Tönen lobt, wusste es. ${ }^{30}$ Eine Frömmigkeit, die den Jubel nicht vergisst, liegt sicher nicht falsch. Und es muss nicht immer Bach sein. In der Kirche darf auch gejazzt oder gejodelt werden. Man muss nur genau hinhören. Wenn man Glück hat, vergisst man die Zeit und meint, dass schon alles glüht, glänzt und hofft. Aber es ist nicht soweit. „Es ist noch nicht getan oder geschehen, es ist aber im Gang und im Schwang. Es ist nicht das Ende, es ist aber der Weg. Es glüht und glänzt noch nicht alles, es reinigt sich aber alles.“ Darum muss die Reformation weitergehen.

Ich schließe mit einem Rat von Bruno Latour, dem bekannten französischen Soziologen, der ein Buch über das Jubilieren mit Tiefgang geschrieben hat:

Vielleicht gibt es wirklich spirituelle Menschen, aber der sicherste Test, sie von den falschen zu unterscheiden, besteht darin, ob sie ihre Gesprächspartner ,nach oben' führen, [...] oder sie im Gegenteil nach und nach herabführen zu Sprechakten, die den Sprecher transformieren, ohne seinen Wissensdurst im geringsten zu schmälern. Wenn sie den Anspruch erheben, [...] Ihnen Geheimnisse zu enthüllen, Sie in Mysterien einzuweihen, Sie zu hehren Sphären zu erheben, meiden Sie sie; aber halten Sie sich an die, die Sie den Rhythmus jener Worte wiederfinden lassen, die zwar keinen Zugang eröffnen, die nirgendwohin versetzen, vor allem nicht weiter und höher, die Sie aber transformieren, Sie selbst, jetzt, da Sie angesprochen sind. ${ }^{31}$

30 Vgl. dazu Ralph Kunz/Felix Moser: Liturgie und Liturgik in der Reformierten Schweiz, in: Pastoraltheologie (PTh) 98 (2009), 157-172.

31 BRUno Latour: Jubilieren. Über religiöse Rede, Berlin 2011, 53. 
Bereitgestellt von | UZH Hauptbibliothek / Zentralbibliothek Zürich 